\title{
Auto-detection of Longitudinal Changes in Retinal Images for Monitoring Diabetic Retinopathy
}

\author{
Deepali A. Godse \\ Ph.D. Research Scholar in Bharati Vidyapeeth \\ Deemed University College of Engineering, Pune, \\ India
}

\author{
Dattatraya S. Bormane, Ph.D \\ University of Pune, India
}

\begin{abstract}
A computer-aided retinal image analysis could provide an immediate detection and monitoring of abnormalities present in the retinal image. It allows to diagnose some retinal diseases prior to specialist inspection. This paper presents automatic system which can aid in the detection and monitoring of diabetic retinopathy (DR). The method proposed here is based on the preliminary automatic registration of retinal images, and the detection of changes in retinal images. This is done by comparing the registered retinal images. A novel algorithm is developed to achieve accurate registration. It ensures that the detected changes reflect only the real changes, and avoids any artifacts associated with the registration procedure itself. The special facts about retinal images are considered while performing image differencing. The present work in this paper is motivated by the need for automated, objective, quantitative approaches to detect the appearance of lesions and to detect longitudinal changes for monitoring DR. This system will considerably reduce the overall workload of ophthalmologists.
\end{abstract}

\section{General Terms}

Image Processing

\section{Keywords}

Diabetic retinopathy, lesions, longitudinal, registration, retinal images

\section{INTRODUCTION}

Study of color retinal images is considered to be the best diagnostic modality available till date as it is reliable, noninvasive and easy to use. It allows recording the diagnostic data and enabling the ophthalmology consultation afterwards. For a particularly long time, automatic diagnosis of retinal diseases from digital fundus images has been an active research topic in the medical image processing community. The retinal analysis is a key field for the early diagnosis of several eye diseases. Large parts of the image analysis process are still done by hand which contribute to increase the workload for the ophthalmologists. Therefore, there is necessity to develop automatic processes that could help them to reduce that workload [1].

DR usually develops gradually and tends to become worse over a number of years. The signs of presence of DR are small red dots (microaneurysms), reddish regions (hemorrhages), white spots (exudates), whitish blobs (cottonwool spots) or newly growing vessels. Fig. 1 (b) shows a case that indicates presence of DR.

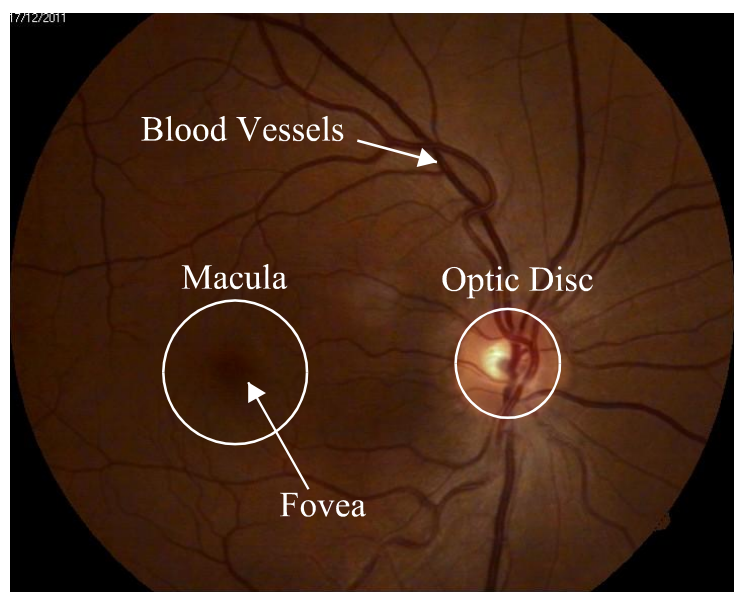

Fig. 1 (a) Normal Retinal Image

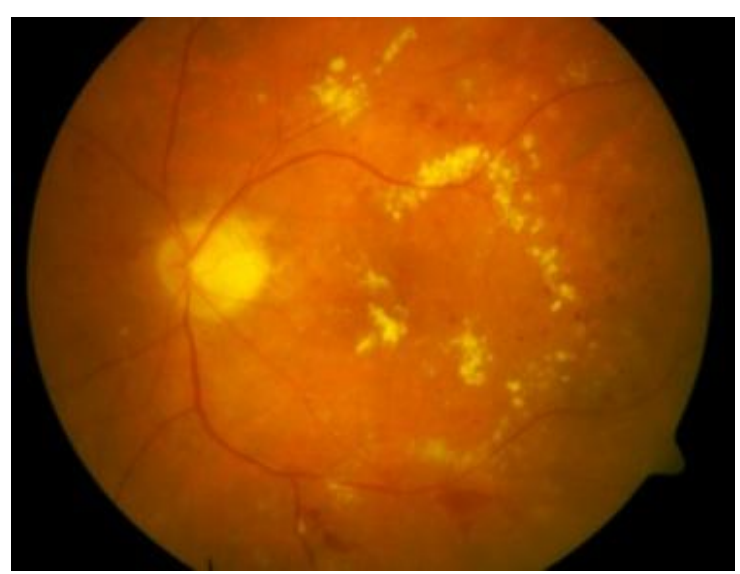

Fig. 1 (b) Retinal Image affected due to DR

Fig. 1 Effect of DR

In today's world, DR is a leading cause of blindness [2]. As the number of people afflicted with diabetes increases worldwide, the need for automated detection methods of DR will increase as well. Early detection can enable timely treatment avoiding further deterioration. The diagnosis of DR can be divided into the following two categories:

1. Screening of the DR

2. Monitoring of the DR 
Most automatic systems approach the detection directly using shape, color, and domain knowledge of DR findings, but the abnormalities can also be found indirectly by detecting changes between two fundus images taken from the same eye in different time moment $[3,4]$. The direct approach contributes to screening of the disease, whereas indirect approach contributes to both screening and monitoring of the DR. Considerable research has been done on segmenting lesions from retinal images using direct approach.

The work in this paper contributes the important element of robust longitudinal change due to presence of DR using indirect approach. The method proposed here is based on the preliminary automatic registration of retinal images of the same diabetic patient acquired over a period of time by a colour fundus camera. The changes that can occur in the retina over time are detected by comparing the registered images. The detection of changes over time are important from a diagnostic standpoint, especially in understanding the dynamic nature of the disease, as well as the response to treatments.

The anatomical features of interest in the retinal image are the blood vessels, optic disk and the fovea as shown in Fig. 1 (a). The blood vessel structures are taken as a relatively stable feature for registration of retinal images [5]. In proposed registration algorithm, derived intensity based features within the optic disk and fovea are used for initial correspondence and bifurcation points of blood vessels are used as landmarks for image registration. There are many possible groups of transformations [6] that can be used to register two images. In this paper a novel technique is proposed for accurate registration of images based on affine transformation model.

The existing change detection techniques can be classified into two categories: pixel-based and region-based methods [7]. In this paper pixel-based image change detection technique based on image differencing followed by thresholding is proposed [8].

\section{MATERIAL AND METHODS}

Table Database used for Auto-detection of longitudinal changes in retinal images is shown in 1 .

Table 1. Database used for change detection

\begin{tabular}{|c|l|c|}
\hline $\begin{array}{c}\text { Sr. } \\
\text { No. }\end{array}$ & \multicolumn{1}{|c|}{ Test Database } & $\begin{array}{c}\text { No. of } \\
\text { Images }\end{array}$ \\
\hline 1 & $\begin{array}{l}\text { Walimbe Eye Clinic, Pune (M.S.), } \\
\text { India }\end{array}$ & 35 \\
\hline 2 & $\begin{array}{l}\text { Bhagali Clinic and Nursing Home, } \\
\text { Pune (M.S.), India }\end{array}$ & 30 \\
\hline 3 & Bharati Hospital, Pune (M.S.), India & 80 \\
\hline
\end{tabular}

Test databases shown in Table 1 are local retinal image databases collected from local ophthalmic clinics in Pune, M.S., India. In addition to this publically available standard retinal image database, DRIVE of 40 images is also used.

Fig. 2 shows the proposed system for auto-detection and monitoring of DR. It mainly involves pre-processing, image registration and change detection.

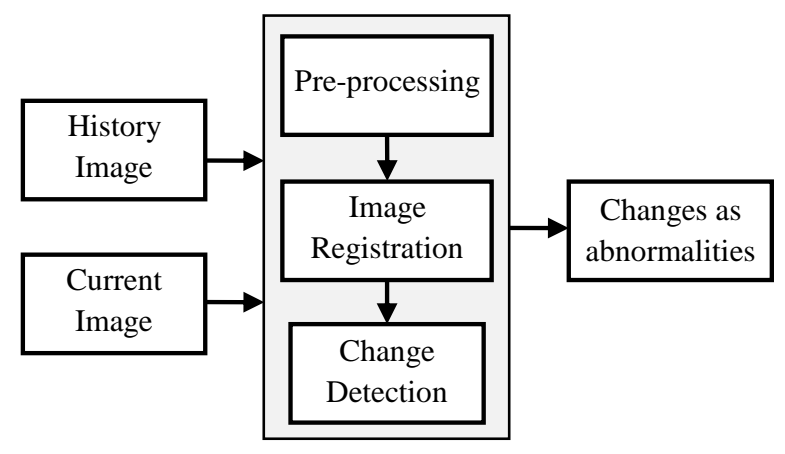

Fig. 2 Stages of Proposed System

\subsection{Pre-processing}

The retinal images in the local database are captured by Kowa VX-10i retinal camera, Japan. The images are of poor quality due to patient's movement, poor focus, bad positioning, reflections, inadequate illumination, non-sufficient contrast, presence of noise, etc. Pre-processing is performed to improve the quality of retinal images. The modified local histogram equalization (LHE) technique is used which provides good contrast enhancement with less time complexity. In modified LHE technique, a local histrogram equalization is applied on a $(71 \times 71)$ window and only the intensity of center pixel of the window is modified with a newly obtained value. Then the window is moved across the image and same procedure is repeated in each case. When window is moved from one position to next one, just one new column or row at the side of window is added and one row or column at another side of the window is omitted. So it is not necessary to re-process pixels which are common in both windows. Only the first window in the image needs to process every pixel in the window. Of course, some boundary conditions need to be checked and resolved during practical implementation.

In modified LHE, the new gray level depends on the cumulative distribution function $(c d f)$ value of the center pixel of a window. The total number of pixels in the window

$(W \times W)$ are fixed, say $W^{2}$ and since gray level mapping in modified LHE is done only for the centre pixel of the current block, the cumulative distribution function $(c d f)$ is derived either by counting pixels having gray level values less than the gray level of center pixel or greater than it. The $c d f$ is derived by counting number of pixels having gray level lower than gray level of centre pixel using equation 1 . On the contrary, when the gray level of centre pixel is higher than $L / 2$, the $c d f$ is derived by counting number of pixels having gray level higher than gray level of centre pixel using equation 2.

$$
\begin{array}{ll}
\text { For } l \leq L / 2 & c d f_{x}(l)=\sum_{i=0}^{l} P(i) \\
\text { For } l \leq L / 2 & c d f_{x}(l)=W^{2}-\sum_{i=l+1}^{L} P(i)
\end{array}
$$

where $P(i)$ is the image's histogram for pixel value $i, l$ is the gray level of the center pixel of the current block and $L$ is the total number of gray levels in the retinal image. Here, we have taken window of $71 \times 71$ pixels (i.e. $W=71$ ). 
The modified LHE technique is faster as compared to traditional histogram equalization technique because it avoids re-processing of common pixels within the two consecutive windows and modified limits for calculating $c d f$ reduce a substantial time of computation.

\subsection{Image Registration}

A necessary pre-processing step for all change detection algorithms is accurate image registration, the alignment of several images into the same coordinate frame. Registration refers to the process of establishing the geometric transformations between images taken at different times, from same or different sensors, or from different viewpoints [9]. Registration is a difficult task in image processing because the correspondence problem is not straightforward. There are different algorithms developed for registration and each generally applies to a specific type of images [10]. Retinal fundus images are challenging to align due to the patientdependent optical characteristics of the eye, coupled with camera [5].

Highly accurate registration is essential for change detection since even modest registration errors result in false indication of change [4]. The similar images are generally misregistered by rotation, scaling and translation. In case of retinal images, the rotational movement is known to be very small (approaching and never exceeding $5^{\circ}$ ). The scaling of one image with respect to the other can be controlled by asking the patient to fixate his eyes, and the head is held to the fundus camera. The translation occurs in the vertical and in the horizontal directions and as there is no detachment, the retina is known to move rigidly [11].

Feature based registration technique is used in proposed system. The bifurcation points of vessels within retinal image are used as control points for registration of two images. The registration involves the steps which are discussed in detail here.

\subsubsection{Bifurcation Points Detection}

Green channel image shows better contrast as compared to red or blue channel image. Therefore, a threshold value is estimated considering green channel histogram $[12,13]$. The thresholding provides a binary image. Then it is thinned using Stentiford algorithm [14].

To locate bifurcation points, the vessels are traced from end points. If a point has a single neighbor, that is marked as end point and if there are more than two neighbors, it is marked as bifurcation point of vessel. Also, continuity of vessels is checked. If there are at least 50 pixels in continuation, those vessels are only considered so that other small segments which are appeared because of noise are removed. To locate the accurate bifurcation points, the entire process is repeated with different sized windows, $21 \times 21$ window, $41 \times 41$ window and $61 \times 61$ window for modified local histogram equalization. The bifurcation points which are common in all three cases are considered as accurate bifurcation points. The bifurcation points are detected in both the retinal images those are to be registered.

\subsubsection{Obtaining corresponding control points in two images which are to be registered}

The accurately detected two bifurcation points spaced by maximum distance are selected as control points in the reference image (history image). They are denoted as A and B. The corresponding points in the sensed image (current image) are obtained using distance criteria between selected bifurcation points, centre of the brightest region in the OD and centre of the darkest region in the macula. For segmentation of OD, macula and locating their intensity based features, algorithms developed in previous work $[15,16]$ are used.

\subsubsection{Affine Transformation}

Unique feature points within image are used as control points for registration in feature based registration method. The optic disc, fovea, blood vessel bifurcations and crosses can be used as control points for registering retinal images [17]. Here, bifurcation points of vascular network are used as control points. The selected control points are $A\left(x_{A}, y_{A}\right)$ and $B\left(x_{B}, y_{B}\right)$ in the reference image and corresponding control points are $A^{\prime}\left(x_{A^{\prime}}, y_{A^{\prime}}\right)$ and $B^{\prime}\left(x_{B^{\prime}}, y_{B^{\prime}}\right)$ in the sensed image respectively.

Scaling factor is determined by taking the ratio of diameters of two retinal images to be registered. If scaling is present, that is removed by considering scaling factor and applying scaling transformation. If only translation is present, that is,

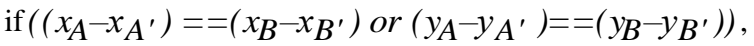
images are registered by translation transformation. If rotation is also involved, it is necessary to determine transformation parameters to perform transformation.

The transformation parameters are obtained by geometrical model as shown in Fig. 3. The proposed method obtains centre of rotation, angle of rotation and direction of rotation from known pair of corresponding control points from two images. Fig. 3 shows geometrical model designed to obtain these details.

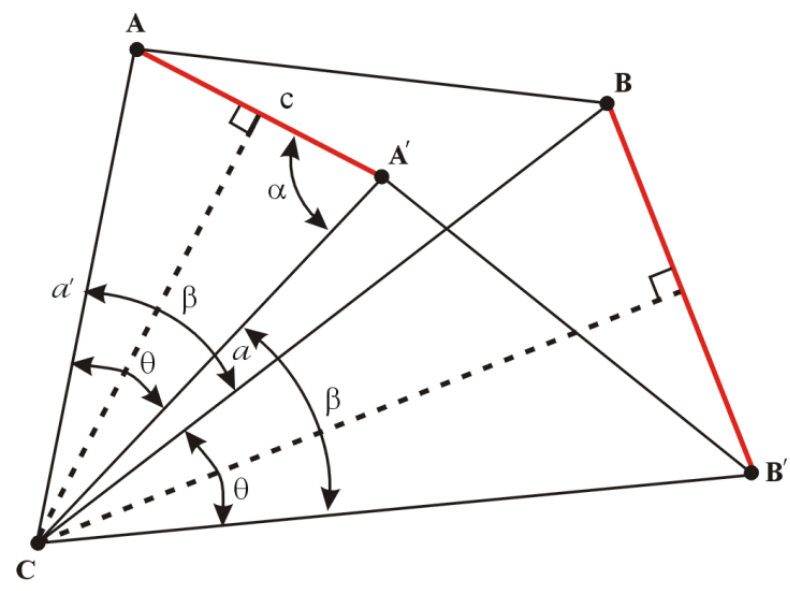

Fig. 3 Geometrical model to determine transformation parameters

\subsubsection{Centre of Rotation}

The intersecting point of perpendicular bisectors of AA' and

$\mathrm{BB}^{\prime}$ is the centre of rotation $C\left(x_{C}, y_{C}\right)$. 


\subsubsection{Angle of rotation}

As shown in Fig. 3, the angle of rotation $\angle \theta$ is obtained using cosine rule,

$$
\begin{gathered}
\cos \alpha=\left(\mathrm{c}^{2}+\mathrm{a}^{2}-\mathrm{a}^{\prime 2}\right) / 2 \mathrm{ca} \\
\sin \angle \theta=\frac{c \sin \alpha}{a^{\prime}} \\
\therefore \quad \theta=\sin ^{-1}\left(\frac{c \sin \alpha}{a^{\prime}}\right)
\end{gathered}
$$

\subsubsection{Direction of rotation}

The slopes of lines $C A$ and $C A^{\prime}$ decide the direction of rotation.

\subsubsection{Transformation}

Once centre of rotation, angle of rotation and direction of rotation are determined, the resultant co-ordinates of every pixel within the image are obtained by applying the transformation matrix for rotation about an arbitrary point

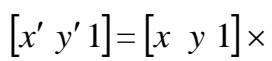

$\left[\begin{array}{ccc}\cos \theta & \sin \theta & 0 \\ -\sin \theta & \cos \theta & 0 \\ -x_{i} \cos \theta+y_{i} \sin \theta+x_{i} & -x_{i} \sin \theta-y_{i} \cos \theta+y_{i} & 1\end{array}\right]$

where $(x, y)$ are original co-ordinates, $\left(x^{\prime}, y^{\prime}\right)$ are resultant co-ordinates and $\theta$ is the angle of rotation.

\subsubsection{Accuracy of Registration}

Here, bifurcation points of vessels are used as control points. Accurate control points detection is very much important step in registration algorithm. The steps taken for accurate bifurcation points detection are as follows:

The Euclidean distances of all detected bifurcation points obtained initially and the points obtained after transformation are used to calculate registration error.

The average of Euclidean distances between original and corresponding transformed points is denoted as error. It is calculated as,

$$
\text { error }=\frac{\sum_{i=1}^{n} D_{i}}{n}
$$

The transformation parameters are obtained by changing selected control points in reference image by inter-pixel distance of \pm 0.1 pixel increment up to 2 pixels in $\mathrm{X}$ and $\mathrm{Y}$ direction. The reference image is transformed using transformation parameters for which error is minimum.

The proposed registration algorithm is tested on 185 retinal image pairs and average error is 0.496 pixel.

\subsection{Change Detection}

Once the images are registered, the changes are detected using change detection algorithm. The major steps in the change detection algorithm are image differencing, thresholding and noise removal.

\subsubsection{Image Differencing}

In retinal images, an optic disc is generally the brightest part within image and maximum intensity variations within the image are usually observed near the optic disc. Considering this fact, the image is divided into two regions. The first region is the region occupied by optic disc and the second region is the region occupied by the remaining portion of the retinal image. The two regions of the image are normalized separately. Since green channel image shows better contrast as compared to red or blue channel image, an optic disc region is normalized considering only green channel intensity.

It is observed that changes due to microaneurysm and hemorrhages reduce the red channel intensity more than the green channel intensity and changes due to hard and soft exudates increase the green channel intensity more than the red channel intensity. Also it is observed that these changes appear in the second region. So, the second region is normalized considering intensity of green channel and intensity of red channel separately. The pixel intensity values in one image are normalized to have the same mean and variance as those in another, i.e.,

$$
I_{2}^{\prime}(x, y)=\frac{\sigma_{1}}{\sigma_{2}}\left(I_{2}(x, y)-\mu_{2}\right)-\mu_{1}
$$

where $\mu_{1}, \sigma_{1}$ and $\mu_{2}, \sigma 2$ are the mean and standard deviation of the intensity values of $I_{1}, I_{2}$ respectively. $I_{2}^{\prime}$ is the normalized image of image $I_{2}$ [4].

After normalization, the mean and standard deviation of the two images are equalized. Hence, the difference image will have zero mean. Mathematically, the difference image is then given by

$$
I_{d}(x, y)=I_{1}(x, y)-I_{2}^{\prime}(x, y)
$$

Alternatively, both images can be normalized to have zero mean and unit variance. This allows the use of decision thresholds that are independent of the original intensity values of the images.

\subsubsection{Thresholding}

To detect the change clearly, it is necessary to apply threshold to $I_{d}(x, y)$ as

$$
T(x, y)= \begin{cases}1, & I_{d}(x, y) \geq \tau \\ 0, & \text { otherwise, }\end{cases}
$$

where the threshold $\tau$ is often determined empirically.

Since the threshold value in equation 10 is important, various automated threshold selection algorithms are proposed. Most of the times, the performance of these algorithms is scene dependent due to the assumptions they are based on. Rosin and Ioannidis [18] investigated the performance of several automated thresholding algorithms using a large set of difference images calculated from an automatically created ground truth database. They give results based on several measures for a complete evaluation. Here, the proposed method takes benefit from two different threshold selection methods. These are Otsu's method [19] and Kapur's method [20]. It is observed that for smaller changes Kapur's method works well. On the other hand, for major changes, Otsu's 
method provides better results. The proposed method selects the maximum of two threshold values derived from these two thresholding methods.

\subsubsection{Noise Removal}

Some noise still remains in the threshold image. This noise is removed using the median filter. It is a nonlinear digital filtering technique, commonly used to remove noise.

\section{RESULTS}

The proposed system is tested on 185 retinal image pairs collected from local ophthalmic clinics in Pune, MS, India and publically available standard DRIVE database. Local database (145 image pairs) includes images captured over a

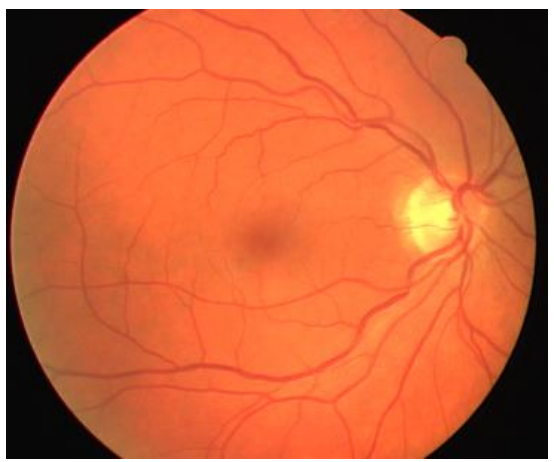

Fig. 4 (a) Reference (history) Image

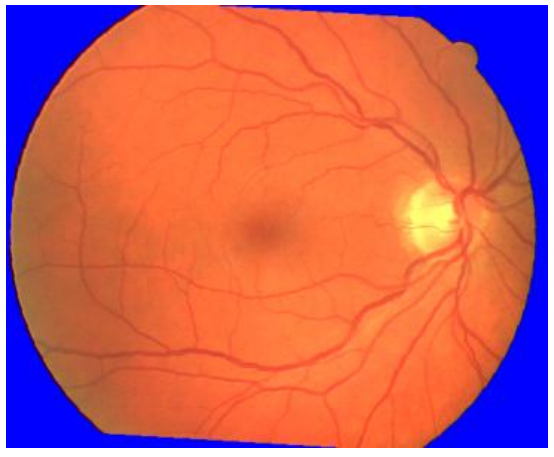

Fig. 4 (c) Registered Reference Image

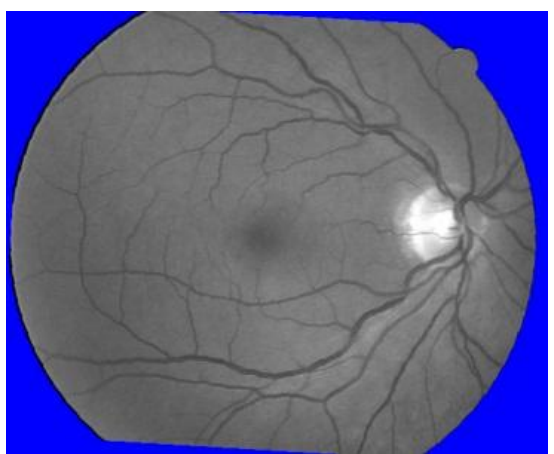

Fig. 4 (e) Grey Level Reference Image period of time. For DRIVE database, 40 retinal images are available and those are considered as history images. The current images are produced by rotating, scaling, translating the history images with different angles, scale factors, translation factors and making DR changes in them under guidance of an ophthalmologist. Fig. 4 (a) and Fig. 4 (b) show the reference image and sensed image, respectively. The registered reference image is shown in Fig. 4 (c). Fig. 4 (d) shows sensed image with mask. Fig. 4 (e) is grey level reference image and Fig. 4 (f) is intensity equalized sensed image. The difference image is shown in Fig. 4 (g). Fig. 4 (h) is output of threshold whereas Fig. 4 (i) is the output of median filter.

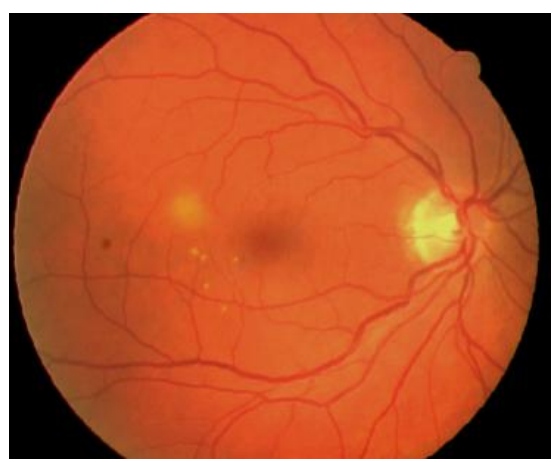

Fig. 4 (b) Sensed (current) Image

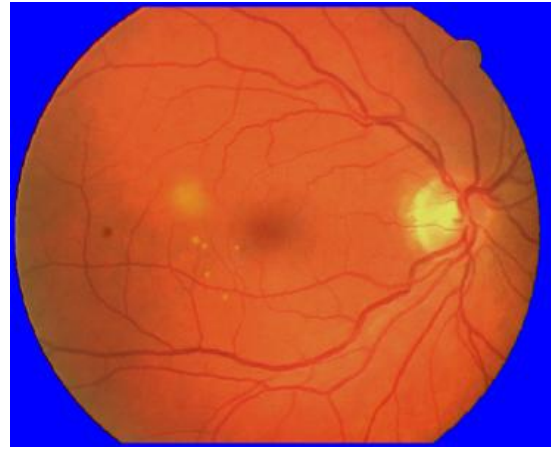

Fig. 4 (d) Sensed Image with mask

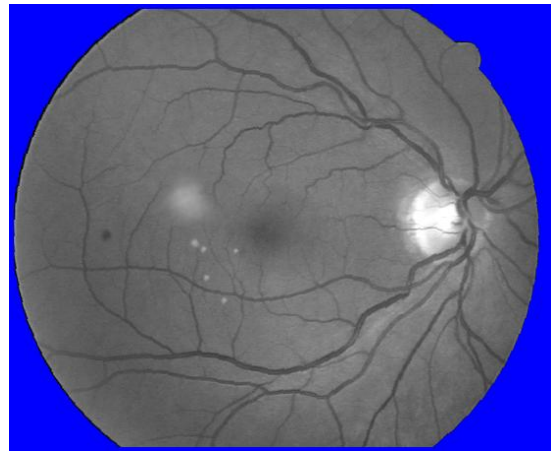

Fig. 4 (f) Intensity equalized Sensed Image 


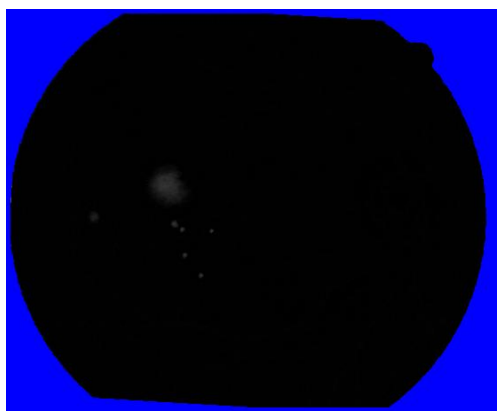

Fig. 4 (g) Difference image

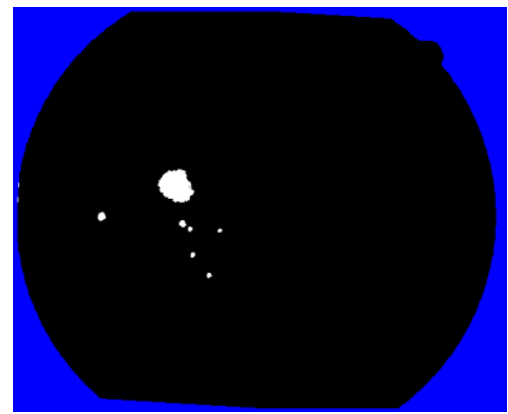

Fig. 4 (h) Threshold image

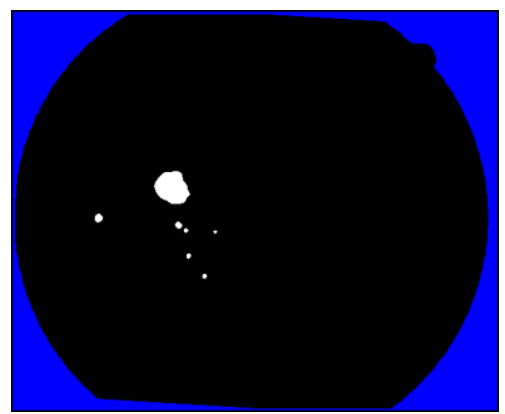

Fig. 4 (i) Median Filtered image

Fig. 4 Output of each stage in overall system

The software is developed for proposed system and the snapshot of it is shown in Fig. 5.

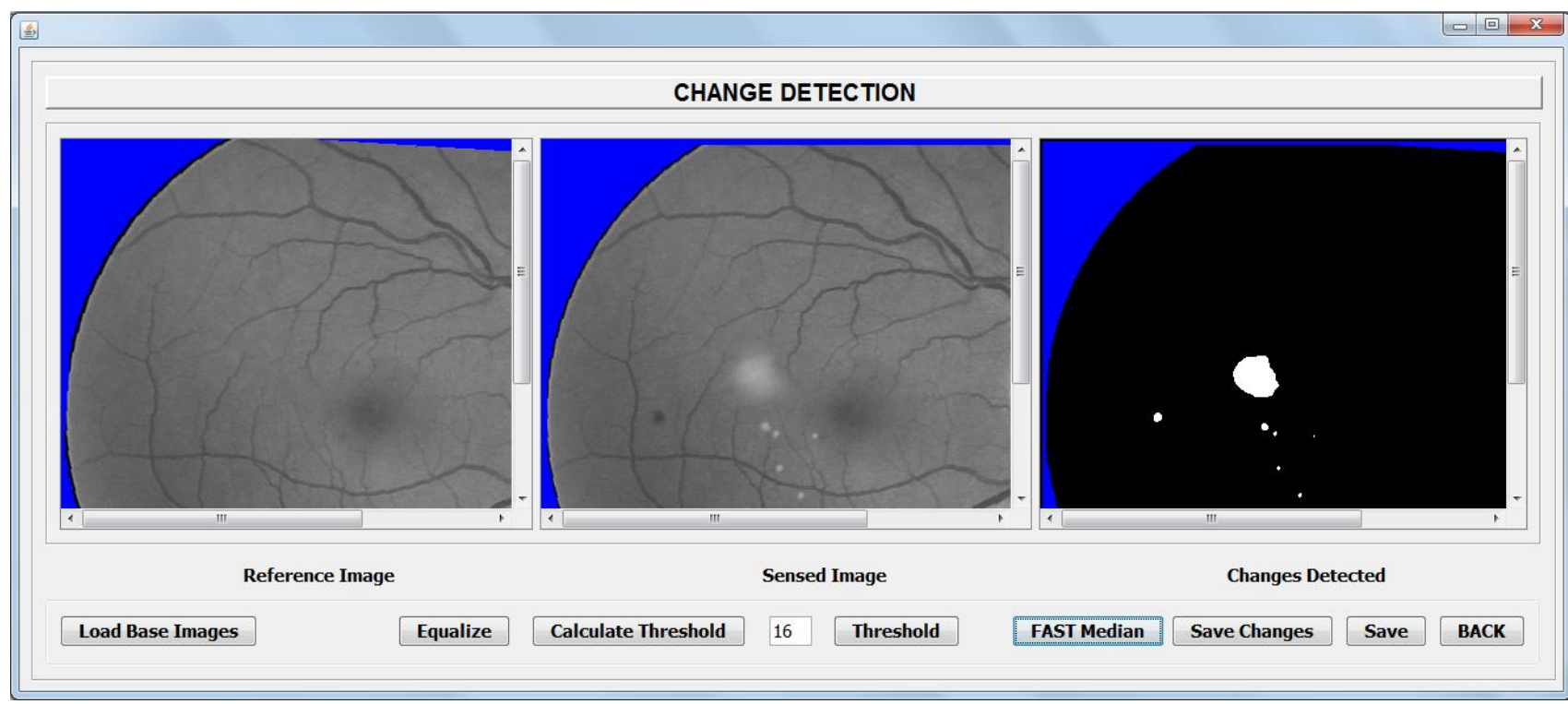

Fig. 5 Snapshot of the proposed system

\section{DISCUSSION}

The proposed system is designed for auto-detection and monitoring of diabetic retinopathy. At each stage algorithms are specially designed to work well for retinal images and changes due to presence of diabetic retinopathy. The method proposed here is based on automatic registration of retinal images, and the detection of the changes that can occur in the retina over time due to presence of DR. A necessary preprocessing step for all change detection algorithms is accurate image registration. Highly accurate registration is required to obtain good change detection results. There is no universal technique for solving all registration problems. The optimal solution is highly dependent on the nature of the image and system requirements. Thus the development of special registration technique for retinal images is appropriate. In this paper an automated feature based method has been presented for registration of retinal images. When feature-based registration is used, the accuracy of the features themselves must be considered in addition to the accuracy of the registration algorithm. So the methods which detect features accurately are used. Special steps are included in the registration algorithm to achieve high accuracy. An affine transformation model is designed to determine transformation parameters needed for registration. The transformation parameters which provide minimum control point error and hence minimum registration error are used for actual registration. The special facts about retinal image anatomy and changes due to presence of DR are considered while performing image differencing step of change detection algorithm. The proposed method is computationally efficient and totally automatic. It also works well on very low quality images.

\section{CONCLUSION}

The work in this paper is mainly useful in ruler areas where super-specialist in ophthalmology are rarely available. The trained technician or expert can collect the retinal images of diabetic patients at those places. The system developed through this work will categorize the collected images as normal and abnormal and only abnormal cases will be sent to super-specialist through internet. This is an addition to currently existing tele-ophthalmology system in which retinal images captured by retinal fundus camera are brought to main centre for further analysis and diagnosis. People with diabetes should have regular eye examinations. If diabetic retinopathy is detected at an early stage, it enables diabetologists to be involved at an earlier and make recommendations to health style changes that could reduce the likelihood of surgery. As 
number of people afflicted due to diabetes are increasing worldwide, there is necessity of such auto-system. The workload of ophthalmologists will considerably be reduced as they have to check only those cases for which change is detected through the proposed system.

\section{ACKNOWLEDGMENTS}

The authors would like to thank Dr. Tejaswini Walimbe, DO, DNB (OPHTH), FAEH, Consultant Eye Surgeon, Glaucoma and Medical Retina Specilist, Walimbe Eye Clinic and Dr. Meenakshi Bhagali, M.S., Eye Specialist, Bhagali Clinic and Nursing Home, Pune for providing retinal image database. The authors would also grateful to Dr. Neelam Puthran, Head of Ophthalmology Dept. and Dr. Deepa Muzumdar, Ophthalmologist, Bharati Hospital, Pune for their valuable guidance and suggestions.

\section{REFERENCES}

[1] F. Lalibert, L. Gagnon and Y. Shaeng, 2002. Registration and fusion of retinal images: A comparative study, Proc. of $16^{\text {th }}$ International conference on pattern recognition, 1 : pp. 715-718.

[2] J. Evans, C. Rooney, S. Ashgood, N. Dattan and R. Wormald, 1996. Blindness and partial sight in England and Wales April 1900-March 1991, Health Trends, vol. 28, pp.5-12.

[3] Harihar Narasimha-Iyer, Ali Can, Bandrinath Roysam, Charles V. Stewart, Howard L.Tanenbau, Anna Majerovics, and Hanumant Singh, June 2006. Robust detection and classfication of longitudinal changes in color retinal fundus images for monitoring diabetic retinopathy, IEEE Transactions on Biomedical Engineering, 53(6):1084-1098.

[4] Richard J. Radke, Srinivas Andra, Omar Al-Kofahi, and Badrinath Roysam, March 2005. Image change detection algorithms: a systematic survey, IEEE Transactions on Image Processing, 14(3):294-307.

[5] Tsai C. L., Stewart C. V., Tanenbaum H. L., Roysam B., 2004. Model-based method for improving the accuracy and repeatability of estimating vascular bifurcations and crossovers from retinal fundus images," IEEE Trans. Inf. Technol. Biomed. 8(2), pp.122-130.

[6] Can A., Stewart C. V., Roysam B., Tanenbaum H. L.,2002. A feature-based, robust, hierarchical algorithm for registering pairs of images of the curved human retina," IEEE Trans. Pattern Anal. Mach. Intell. 24(3), pp. 347-364.

[7] Liyuan Li and Maylor K. H. Leung, February 2002. Integrating Intensity and Texture Differences for Robust Change Detection, IEEE Transactions on Image Processing, Vol. 11, No. 2.

[8] Murat İlsever, Cem Ünsalan, May 2012. TwoDimensional Change Detection Methods, Springer, ISBN 978-1-4471-4254-6.
[9] L. G. Brown, , Dec. 1992. A survey of image registration techniques, ACM Comput. Surveys, vol. 24, no. 4, pp. 325-376.

[10] F. Zana, J.C. Klein, May 1999. A multimodal registration algorithm of eye fundus images using vessels detection and Hough transform, IEEE transactions on medical imaging, vol. 18 , no. 5 .

[11] Jian Chen, R. Theodore Smith, Jie Tian, Andrew F. Laine, , 2008. A novel registration method for retinal images based on local features, Conf Proc IEEE Eng Med Biol Soc., pp. 2242-2245.

[12] Lili Xu and Shuqian Luo, , February 2010. A novel method for blood vessel detection from retinal images, School of Biomedical Engineering, Capital Medical University, Beijing, China, doi:10.1186/1475-925X-914.

[13] Chin-Chen Chang, Chia-Chen Lin, Pei-Yan Pai and YenChang Chen, , 2009. A Novel Retinal Blood Vessel Segmentation Method Based on Line Operator and Edge Detector, Proceedings of the Fifth International Conference on Intelligent Information Hiding and Multimedia Signal Processing, IEEE Computer Society Washington, DC, USA, pp. 299-302.

[14] Stentiford F. W. M. and Mortimer R. G., 1983. Some new heuristics for thinning binary hand-printed characters for OCR", IEEE Trans. on systems, Man. and Cyb. 13, no.1, pp. 81-84

[15] Deepali A. Godse and Dr. Dattatraya S. Bormane, Nov 2012. Automated localization of centre of optic disc and centre of macula in retinal images, CiiT International Journal of Biometrics and Bioinformatics, vol. 4, no. 16 , pp. 896- 901 .

[16] Deepali A. Godse and Dr. Dattatraya S. Bormane, 2013. Automated Localization of Optic Disc in Retinal Image, International Journal of Advanced Computer Science and Applications, vol. 4, no. 2.

[17] Winston Li and Henry Leung, August 2004. A Maximum Likelihood Approach for Image Registration Using Control Point And Intensity, IEEE Transactions On Image Processing, vol. 13, no. 8.

[18] P. Rosin and E. Ioannidis, October 2003. Evaluation of global image thresholding for change detection, Pattern Recognition Letters, vol. 24, no. 14, pp. 2345-2356.

[19] N. Otsu, January 1979. A threshold selection method from gray-level histograms, IEEE Transactions on Systems. Man, and Cybernetics, vol. 9, pp 62-66.

[20] J. N. Kapur, P. K. Sahoo and A. K. C. Wong, 1985. A new method for grey-level picture thresholding using the entropy of the histogram", Computer vision, graphics and image processing, vol. 29, pp. 273-285. 